\title{
Extracranial oral cavity metastasis from glioblastoma multiforme: A case report
}

\author{
PHILIPP GÜNTHER KUP ${ }^{1,2}$, CARSTEN NIEDER $^{3}$, GUIDO WINNEKENDONK ${ }^{4}$, \\ IRENÄUS ANTON ADAMIETZ ${ }^{1,2}$ and KHASHAYAR FAKHRIAN ${ }^{1,2}$
}

\author{
${ }^{1}$ Department of Radiation Oncology, Marien Hospital Herne, Ruhr University Bochum, \\ D-44625 Herne; ${ }^{2}$ Department of Radiation Oncology, St. Josef Hospital Bochum, Ruhr University Bochum, \\ D-44791 Bochum, Germany; ${ }^{3}$ Department of Oncology and Palliative Medicine, Nordland Hospital, 8005 Bod $\varnothing$, \\ Norway; ${ }^{4}$ Department of Radiology, Marien Hospital Herne, Ruhr University Bochum, D-44625 Herne, Germany
}

Received December 18, 2015; Accepted June 1, 2016

DOI: $10.3892 / \mathrm{mco} .2016 .991$

\begin{abstract}
Glioblastoma multiforme is the most common primary malignant brain tumor. The clinical outcome following diagnosis remains extremely poor. The treatment of choice is wide surgical resection of the visible tumor, frequently followed by adjuvant combined radiochemotherapy (RCTx) with temozolomide as the chemotherapeutic agent. Extracranial metastases are extremely rare, with $<200$ cases of extracranial metastases from glioblastoma multiforme reported in the literature to date. We herein present a case of a patient suffering from a fast-growing metastasis to the oral cavity, completely filling the buccal cavity within 2 weeks, as the only manifestation of recurrent glioblastoma multiforme following initial surgical resection and adjuvant RCTx.
\end{abstract}

\section{Introduction}

Glioblastoma multiforme is a highly malignant glioma of astrocytic origin (1). The tumor is defined as grade IV according to the World Health Organization (WHO) classification, and is a cytologically malignant, mitotically active, necrosis-prone neoplasm, typically associated with rapid preand postoperative disease evolution and a fatal outcome, with a median survival of $\sim 12$ months $(2,3)$.

With an incidence of 3.19/100.000 patient years, glioblastoma multiforme is the most common glioma in adults (typical age, 50-70 years), located mostly in the frontotemporal cerebral lobes $(1,4)$.

Correspondence to: Dr Khashayar Fakhrian, Department of Radiation Oncology, Marien Hospital Herne, Ruhr University Bochum, 40 Hoelkeskampring, D-44625 Herne, Germany

E-mail:khfmed@yahoo.com

Key words: glioblastoma multiforme, extracranial metastases, oral cavity metastasis
The treatment of choice currently consists of maximal safe surgical resection and postoperative concomitant radiochemotherapy ( $\mathrm{RCTx}$ ), followed by further chemotherapy with the orally administered alkylating drug temozolomide (5). The indication for radiotherapy (RTx) depends on the patient's general condition [Karnofsky performance status (PS) or Eastern Cooperative Oncology Group PS], age and extent of surgery (6,7). Typically, conformal RTx consists of 54-60 Gy (1.8-2.0 Gy per fraction), with limited safety margins of a maximum $2.0 \mathrm{~cm}$. Long-term local control is difficult to achieve. Extracranial metastases are reported to occur in 0.4-0.5\% of all cases (8-12). In 1928, Davis first reported a patient with disseminated glioblastoma (13). The affected organs included the lung, upper extremity soft tissue and chest wall. The natural history of glioblastomas that metastasize to organs outside the central nervous system is largely unknown. Between 1928 and 2009, 88 cases of extracranial metastasis from glioblastoma $(n=83)$ and gliosarcoma $(n=5)$ have been published, reported and analyzed by Lun et al (14). In 2015, Pietschmann et al reviewed existing data and performed a new meta-analysis with 150 cases (15). In addition to brain stem and spinal axis, lymph nodes and visceral organs, including the liver, lung, pleura and bones, were found to be involved. In only one of all documented cases an extracranial oral cavity metastasis of significant extent and exhibiting rapid growth was described (16).

\section{Case report}

A 70-year-old female patient was admitted to our hospital suffering from progressive lack of concentration and cognitive deficit. The patient's husband also reported a personality change. Arterial hypertension, hyperthyroidism and lipometabolic disorder were documented, without other comorbidities. Initially, we performed magnetic resonance imaging (MRI) of the head, which revealed a massive tumor in the right frontal lobe, with perifocal oedema and midline dislocation of $6 \mathrm{~mm}$, suspicious for glioblastoma (Fig. 1). The tumor was sized $4.2 \times 2.8 \times 3.9 \mathrm{~cm}$. Navigated maximal safe tumor excision was performed with osteoplastic craniotomy and dura mater plastic surgery. The postoperative course was 
uneventful. Histology and immunohistochemical investigation revealed a $\mathrm{O}^{6}$-methylguanine-DNA methyltransferase-positive glioblastoma multiforme, WHO grade IV. Adjuvant RCTx was initially performed with low-dose temozolomide $140 \mathrm{mg} /$ day, subsequently escalated to the standard dose of $200 \mathrm{mg} /$ day (total dose 60 Gy, 2 Gy per fraction).

One year after the diagnosis, the patient developed a rapidly progressive oral cavity tumor, filling the entire buccal cavity within 2 weeks (Fig. 2). Following biopsy, the histological examination revealed a necrotic retromaxillary metastasis of the previously diagnosed glioblastoma, exhibiting histological characteristics identical to those of the primary resected tumor. Systemic metastases were not identified. The patient was unable to speak or eat (Fig. 3). Following extensive interdisciplinary discussion, we decided to proceed with local palliative RTx and supportive measures. The patient developed massive breathing difficulties, necessitating transfer to the intensive care unit, followed by tracheotomy and immediate mechanical ventilation. We first planned to administer a total of $45 \mathrm{~Gy} / 3 \mathrm{~Gy}$ per fraction. In the light of rapid progress of the tumor, a dose of 5 Gy per fraction was applied (Fig. 3). However, after 4 applications, the patient's relatives requested discontinuation of RTx and the patient succumbed to the disease soon thereafter.

\section{Discussion}

We herein report the case of a patient suffering from glioblastoma with subsequent retromaxillary metastatic spread completely filling the buccal cavity. Initial treatment with cranial tumor extirpation followed by RCTx with 60 Gy and temozolomide was performed. At the time of relapse and clinical deterioration, the patient was treated with 20 Gy to the metastatic area. However, RTx was discontinued and the patient succumbed to the disease soon thereafter. Extracranial metastasis from glioblastoma remains an uncommon finding (15). Thus far, a massive metastasis completely filling the buccal cavity has only been reported in 1996 by Horiuchi et al (16) in a 41-year-old female. In contrast to our patient, simultaneous local recurrence of the tumor was described. MRI and computed tomography scans revealed orbital, nasal and oral masses extending inferiorly from the recurrent tumor in the right temporal lobe through the anterior and middle skull base. The oral cavity tumor and swelling of the patient's face grew rapidly. The common characteristics of the two cases are rapid growth and gender of the patient, but no connection to cranial tissue was radiologically detected in our patient.

The reasons for such extensive spread in this particular location and the growth rate are not well understood. A necessary requirement for extracranial metastasis is tumor cells crossing the dura mater, which is the most important barrier. Surgery and radiation therapy may lead to dural damage and potentially facilitate extracranial extension. In our case, no lesion of the dura mater was documented during surgery. A ventricular systemic shunt was not indicated. Our 70-year-old patient underwent craniotomy followed by RTx with simultaneous chemotherapy. Younger patients exhibit an increased risk of extraneural involvement due to prolonged survival $(15,17)$. The most common sites of extra-

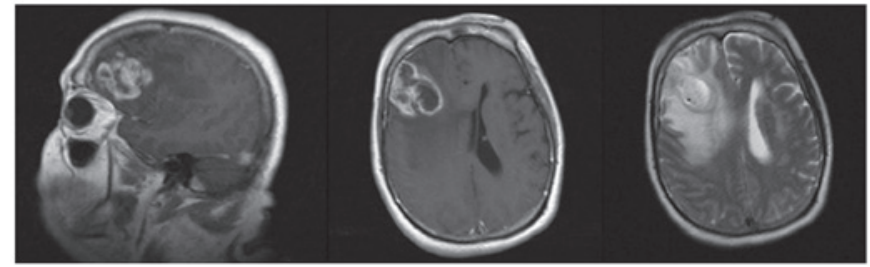

Figure 1. Preoperative magnetic resonance imaging. Left/middle: T1-weighted axial gadolinium-enhanced image showing an enhancing tumor of the right frontal lobe. Right: T2-weighted image showing the same lesion as in the previous images, with notable edema.

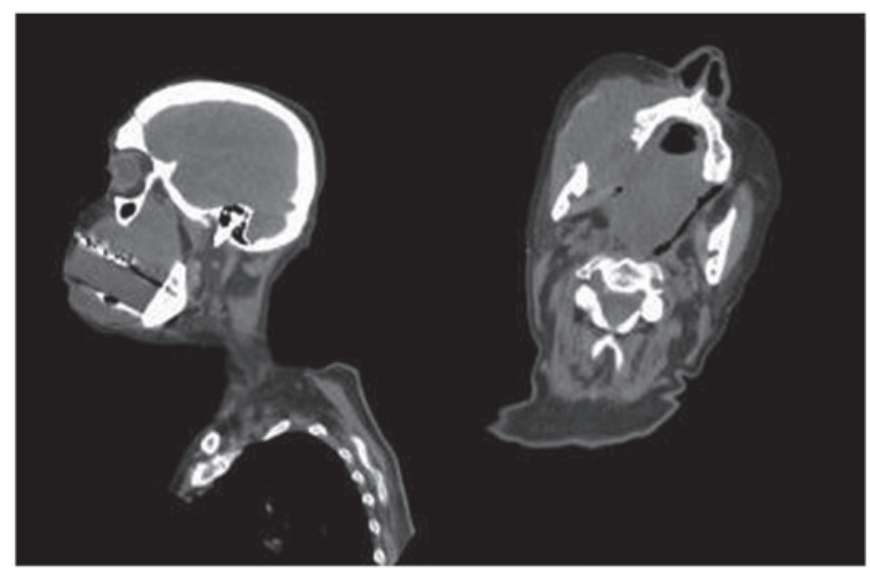

Figure 2. Computed tomography of the head performed during the second clinical presentation of the patient. A mass is identified in the right buccal space, without extension outside the skull base.

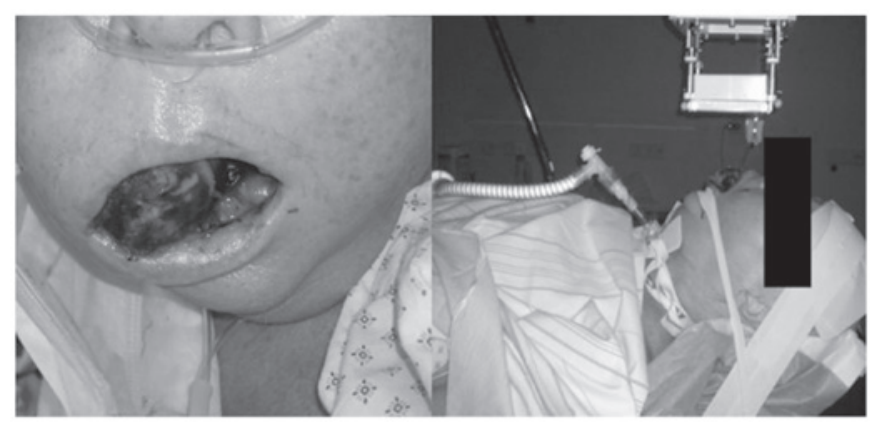

Figure 3. Left: Visible tumor mass in the right buccal cavity. Right: Picture of the patient during radiotherapy; the tumor mass is seen emerging from the buccal cavity.

cranial metastases from glioblastoma are the lungs, bone (bone marrow), lymph nodes and soft tissues (14). This is the second report of an extracranial metastasis with a retromaxillary localization, exhibiting rapid growth and completely filling the buccal cavity. Glioblastoma is a tumor with fatal outcome. Although extracranial metastases remain extremely uncommon, extraneural spread of the tumor is possible and must be considered in symptomatic patients during follow-up. Extracranial glioblastomas may present with an extraordinarily rapid progression pattern, as in our case. Therefore, fast decision-making and treatment initiation are crucial for optimal palliative treatment. Further investigation is required to improve therapy and outcome. 


\section{References}

1. Louis DN, Perry A, Burger P, Ellison DW, Reifenberger G, von Deimling A, Aldape K, Brat D, Collins VP, Eberhart C, et al; International Society Of Neuropathology - Haarlem: International Society of Neuropathology - Haarlem consensus guidelines for nervous system tumor classification and grading. Brain Pathol 24: 429-435, 2014.

2. Krex D, Klink B, Hartmann C, von Deimling A, Pietsch T, Simon M, Sabel M, Steinbach JP, Heese O, Reifenberger G, et al: Long-term survival with glioblastoma multiforme. Brain 130: 2596-2606, 2007.

3. Visser O, Ardanaz E, Botta L, Sant M, Tavilla A and Minicozzi P; EUROCARE-5 Working Group: Survival of adults with primary malignant brain tumours in Europe; Results of the EUROCARE-5 study. Eur J Cancer: September 5, 2015 (Epub ahead of print). doi: 10.1016/j.ejca.2015.07.032.

4. DeAngelis LM: Brain tumors. N Engl J Med 344: 114-123, 2001

5. Preusser M, de Ribaupierre S, Wöhrer A, Erridge SC, Hegi M, Weller M and Stupp R: Current concepts and management of glioblastoma. Ann Neurol 70: 9-21, 2011.

6. Dolecek TA, Propp JM, Stroup NE and Kruchko C: CBTRUS statistical report: Primary brain and central nervous system tumors diagnosed in the United States in 2005-2009. Neuro Oncol 14 (Suppl 5): v1-49, 2012.

7. Stummer W, Reulen HJ, Meinel T, Pichlmeier U, Schumacher W, Tonn JC, Rohde V, Oppel F, Turowski B, Woiciechowsky C, et al: Extent of resection and survival in glioblastoma multiforme: Identification of and adjustment for bias. Neurosurgery 62: 564-576; discussion 564-576, 2008.

8. Smith DR, Hardman JM and Earle KM: Metastasizing neuroectodermal tumors of the central nervous system. J Neurosurg 31 : 50-58, 1969.

9. Pasquier B, Pasquier D, N'golet A, Panh MH and Couderc P: Extraneural metastases of astrocytomas and glioblastomas: Clinicopathological study of two cases and review of literature. Cancer 45: 112-125, 1980 .
10. Astner ST, Pihusch R, Nieder C, Rachinger W, Lohner H, Tonn JC, Molls M and Grosu AL: Extensive local and systemic therapy in extraneural metastasized glioblastoma multiforme. Anticancer Res 26: 4917-4920, 2006.

11. Elena A, Melina C, Raffaele N, Carlo B, Vittoria N, Francesco A, Gaetano F and Marica E: Extraneural metastases in glioblastoma patients: Two cases with YKL-40-positive glioblastomas and a meta-analysis of the literature. Neurosurg Rev 39: 37-46, 2016.

12. Ray A, Manjila S, Hdeib AM, Radhakrishnan A, Nock CJ, Cohen ML and Sloan AE: Extracranial metastasis of glioblastoma: Three illustrative cases and current review of the molecular pathology and management strategies. Mol Clin Oncol 3: 479-486, 2015.

13. Davis L: Spongioblastoma multiforme of the brain. Ann Surg 87: 8-14, 1928.

14. Lun M, Lok E, Gautam S, Wu E and Wong ET: The natural history of extracranial metastasis from glioblastoma multiforme. J Neurooncol 105: 261-273, 2011.

15. Pietschmann S, von Bueren AO, Kerber MJ, Baumert BG, Kortmann RD and Müller K: An individual patient data meta-analysis on characteristics, treatments and outcomes of glioblastoma/gliosarcoma patients with metastases outside of the central nervous system. PLoS One 10: e0121592, 2015.

16. Horiuchi T, Osawa M, Itoh N, Kobayashi S, Nitta J and Hongo K: Extradural extension of glioblastoma multiforme into the oral cavity: Case report. Surg Neurol 46: 42-46, 1996.

17. Bertolini F, Zunarelli E, Baraldi C, Valentini A, Del Giovane C, Depenni R, Falasca A, Giacobazzi P, Malagoli M, Meletti S, et al: Survival in patients with newly diagnosed conventional glioblastoma: A modified prognostic score based on a single-institution series. Tumori 98: 756-761, 2012. 